\title{
Evaluation of Osseo Integrated Implants in Regenerated Jaw Bones after Cyst Enucleation
}

\author{
Dr Richi Burman ${ }^{1}$, Dr Md Rabiul Islam², Dr Anuj Sharma ${ }^{3}$ \\ ${ }^{I}$ (Senior Lecturer, Dept of Oral \& Maxillofacial Surgery, Guru Nanak Institute of Dental Sciences \& Research, \\ India) \\ ${ }^{2}$ (Final yr PGT, Dept of Prosthodontics, Guru Nanak Institute of Dental Sciences \& Research, India) \\ ${ }_{3}^{3}$ (Senior Resident, Dept of Dentistry, Hamdard Institute of Medical Sciences \& Research, India)
}

\begin{abstract}
Aims \& Objective: To evaluate if the implants can be osseointegrated in regenerated bone of patients who had large bony defects following odontogenic cyst enucleation.

Material \& Methods: A total of five patients were chosen for the study, implants were placed one year after surgical cyst enucleation The patients were assessed clinically and radiologically periodically i.e. every month, till 6 months after placement of implant. The success criteria included absence of any sign of periimplant inflammation, clinical mobility of the implant and abutment and finally absence of any peri implant radiolucency when seen on the radiograph.
\end{abstract}

Results: A total of 15 implants were placed in five patients having regenerated bone with $93.3 \%$ osseointegration. One patient showed failure i.e. implant mobility fifteen days after placement. The failure was attributed to poor quality of regenerated bone.

Conclusion: By the findings of this study, we can safely conclude that implants can be successfully osseointegrated in regenerated bone, however poor quality of regenerated bone is the main cause of failure. However studies with larger sample size and concurrent HBO therapy to accelerate bone healing can be done in future.

Keywords: Odontogenic cyst, Regenerated bone, Dental implants

\section{Introduction}

Pathologies of the jaws are extremely distressing even after successful surgery due to the nature of the defects and the effect it has on the patient's esthetics and function. Thereafter the insurmountable challenge it poses to the prosthodontist for rehabilitation of the patient. Conventional dentures were either impossible to fabricate or are quite useless. But over the past two decades rapid advances in material science and refinement of surgical techniques have resulted in extremely reliable titanium implants. These have almost always been the answer for the more complex of dento-alveolar and even extra-oral defects

Though dental implants have been successful means of rehabilitating patients with tooth loss, studies reported in the literature on the success of osseointegration of implants in regenerated bone have been relatively few and none of them have been done in the Indian scenario. ${ }^{1-3}$ The ultimate test of regenerated bone is its amenability to function normally. ${ }^{4}$ Studies that describe osseointegration of implants in bone which is regenerated in large volume bony defects as in cases of cysts and tumors are few.

Here we have conducted a single arm study wherein a total of 15 implants have been placed in regenerated bone of five patients who had large mandibular defects as a result of surgical enucleation of intra bony cysts.

\section{Material \& Methods}

A total of six patients were chosen for the study, which required placement of implants within regenerated bone. The bone regeneration had occurred after enuleation of odontogenic cysts . A time gap of a minimum of 1 year was taken between primary surgery and placement of implants for bone regeneration to occur. No additive bone graft or Platelet Rich Fibrin was added to the cystic cavity to fasten healing. Patients with poor oral hygiene, systemic disease affecting healing and chronic smokers were excluded from the study. The patients were informed of the study and their permission was sought for their participation in the study. An informed consent was obtained from each of the participating patients. The implants were of an Israel Based System called HITECH DENTAL IMPLANTS.

Before placing the implants, a thorough assessment of the patient was done for implant reconstruction that included a detailed history, intraoral and extra oral examination, panoramic radiographs as well as bone mapping. Panoramic radiographs were used for screening examinations and treatment planning of the patients to get a broad perspective of the inferior alveolar canal, maxillary sinus, mental foramina, and nasal floor; and were used to assess the depth of the bone available. 
Since the panoramic films generally have a magnification factor of about $25 \%$, this was anticipated and eliminated during the patient work-up to gain a better appreciation of the actual position of vital structures and the size of implant to be selected. The OPG s were standardized by using known diameter stainless steel shots incorporated in a vacuum-formed stent in the area of proposed implant site worn by the patient at the time of radiography.

The distortion was calculated by comparing the actual diameter of the steel shots with the radiographic diameter as

Distortion $=$ Normal diameter of the steel shot $\quad x \quad 100$

Radiographic diameter

Bone mapping was done for assessment of transverse thickness of bone, the procedure involves anesthetizing the proposed area of the patient and marking the depth of the soft tissue over the alveolar bone using a sterile file at various levels and then marking the recorded depth of the bone over a sectioned cast of the patient.

\section{Surgical Technique}

All patients were operated under local anesthesia. Standard conditions of asepsis and sterility were strictly adhered to during the implant placement procedures The surgical incision was made on the crest, with vertical releasing curvilinear incisions flaring into the vestibule in order to keep the base of the flap wider than the crestal incision width. Full-thickness subperiosteal labial and palatal flaps were reflected to expose the crest and to provide visualization of the vertical cortices of bone. The incision ensured adequate buccal and lingual or palatal attached tissue on either side.

Surgical stents having guide channel were used to place the implant in the correct position so that the implants were placed with their axis parallel to the occlusal forces, with the emergence of the implant angling to meet the functional cusp of the opposing teeth.

Once the alveolar bone to receive the implant was exposed a flat implant bed was prepared using a $1 \mathrm{~mm}$ straight fissure bur wherever it was found necessary. Once the implant site was prepared a surgical guide or stent was placed intra orally and a small round bur or spiral drill was used to mark the implant site. The stent was then removed and the site was checked for appropriate facio- lingual and mesio-distal positioning. If there was an obvious crestal defect then a slight modification of the position was made. The site was then marked to a depth of 1 to $2 \mathrm{~mm}$ perforating the cortical bone using a small round bur. A pilot drill usually $2 \mathrm{~mm}$ in diameter was then drilled in the marked implant site to establish the depth and axis of implant recipient site. The drills were used in a reduction gear hand piece along with a physio-dispenser enabling internal as well as external irrigation to prevent excessive heat generation.

In all cases the drill was used at the speed of 800 to $1000 \mathrm{rpm}$ with copious irrigation. Once the drill holes were made paralleling pins were used to check the parallelism of the drill holes to the adjacent teeth as well as adjacent implant recipient sites, in case multiple implants were being placed. These paralleling pins were used at each stage of surgery to ensure that the axis of the recipient site was not changed. Following the pilot drill, drills with gradually increasing diameters were used to enlarge the implant recipient site till the desired diameter corresponding to the implant diameter was reached. Implants were then placed into the prepared site using a wrench. 3-0 vicryl sutures were placed to close the surgical wound. The patients were monitored on a periodic basis both clinically and radiologically.

The patients were assessed clinically and radiologically periodically. This included assessments post operatively on a monthly basis for six months.

The success criteria include:

1. Absence of any sign of peri implant inflammation or infection.

2. Absence of any clinical mobility of the implant.

3. Absence of any peri implant radiolucency when seen on the radiograph.

\section{Results}

In our study we have made an attempt to evaluate the success of implants in bone which has been regenerated in large bony defects of the jaws such as caused by odontogenic cysts. The study involved placing 15 implants in regenerated bone in five of our patients. All the patients had a history of surgical enucleation of cysts of the jaws and in all the cases bone had regenerated sufficiently into the bony defects. The regeneration of the bone was spontaneous and was not accelerated by any adjuncts. Implants were placed in regenerated bone of such patients under local anesthesia with antibiotic cover in sterile conditions. All cases were followed up for six months to one year. 
During the follow-ups each implant was checked for clinical and radiological signs of implant failure such as infection of peri implant tissue, implant mobility and presence of radiolucent line around the implant when seen on radiograph.

All the implants were exposed between the fourth to sixth months and were checked for clinical mobility once the healing caps were placed. The gingival healing caps were left in situ for 2 to 3 weeks to give time for the gingival collar to form around the implant. Once the gingival healing was complete the healing caps were replaced with abutments for the respective implants. At the time of exposure and further follow-ups there was no evidence of peri-implant infection, and there was no clinical mobility of the implants and follow up radiographs showed no periimplant radiolucency indicating excellent osseointegration.

However, one implant in Case 4 developed clinical implant mobility within 15 days itself, and was considered as failure.

There was no significant surgical morbidity associated with any of the patient receiving implants in regenerated bone apart from Case 4.. Post operatively one of the patients who developed infection or had implant rejection, could be attributed to the poor quality of quality of recipient bone. Henceforth there was $93.3 \%$ osseointegration in regenerated bone in our cases.

TABLE 1 : Showing site, number and result of placing Implants

\begin{tabular}{|r|r|r|r|c|}
\hline Sl no & Site & $\begin{array}{r}\text { No. of Impla } \\
\text { placed }\end{array}$ & $\begin{array}{r}\text { Successful } \\
\text { Osseo } \\
\text { integration }\end{array}$ & $\begin{array}{c}\text { Mobility and } \\
\text { failure of implant }\end{array}$ \\
\hline Case 1 & $37,38,46,47,48$ & 4 & 4 & Nil \\
\hline Case 2 & $42,42,43,44$. & 2 & 2 & Nil \\
\hline Case 3 & $31.32,33,34,35,41$ & 4 & 4 & Nil \\
\hline Case 4 & $34,35,36$ & 3 & 2 & $\begin{array}{c}\text { Implant placed } \\
\text { in 34 region } \\
\text { After 15 days. }\end{array}$ \\
\hline Case 5 & $45,46,47$ & 2 & 2 & Nil \\
\hline
\end{tabular}

V. Discussion

Tumors and cysts of the head and neck region require extensive ablation of the bone and soft tissues. Surgical procedures such as cyst enucleation or tumor resection lead to residual cavities in the bone. These cavities spontaneously get filled by regenerated bone over a period of time. Micro vascular techniques and bone grafts or bone substitutes and bone regeneration through guiding membranes and bone regenerated through distraction osteogenesis may decrease the time of healing and increase the predictability of the results. . Chiapasco et ${ }^{5}$ al studied the rate of spontaneous bone regeneration of defects secondary to enucleation of large ( $>40 \mathrm{~mm}$ ) cysts of the mandible. Near total regeneration of bony defects (mean 81.30\%) occurred at 24 months without any use of bone graft or bone substitute.

Several materials, autogenic and allogenic, have been used to promote and accelerate healing of bone. The principal drawback of bone grafts or free flaps is the need for a second procedure and the attendant morbidity ${ }^{6}$. Fillers like hydroxyapatite act only as a scaffold and have a fairly high rate of infection.

Another technique, which is being extensively studied is Hyperbaric oxygen therapy. Hyperoxia, has potential benefits of improved angiogenesis and neocellualrity. The formation of collagen matrix is essential for angiogenesis and is inhibited by hypoxia. In irradiated tissue hyperbaric oxygen is more effective than normobaric oxygen at raising tissue partial pressure of oxygen and promoting angiogenesis and wound healing. The healing process may also be helped in non-irradiated tissues with compromised perfusion. In all the cases in this study hyperbaric oxygen was used to assist the regeneration of bone.

Ahmed et $\mathrm{al}^{7}$, in their study of healing in calvarial bone defects in rabbits have concluded that HBO therapy greatly enhances the rate of healing of surgical defects. They report $57.64 \%$ of new bone formation with HBO treated rabbits as compared to $23.86 \%$ in defects allowed to heal spontaneously.

All these above-mentioned techniques along with spontaneous bone regeneration have to a large extent, answered the questions posed by bony segments or defects. But the ultimate rehabilitation is the complete restoration of dental and oral function without which the treatment of the patient can be considered incomplete 
Dental implants currently are the closest thing technology has to offer in lieu of a natural tooth. They have performed exceptionally well in restoration of teeth lost in a natural bony arch. The efficacy of dental implants in regenerated bone is yet to be conclusively proved, and there are relatively very few studies which describe the response of regenerated bone towards titanium implants and most of them involve small areas of regenerated bone regenerated by techniques like guided bone regeneration.

The quality of bone in in which the implants are placed has a definite role on its long-term success. Tumor resection or cyst enucleation causes large bone defects. The bone regenerated in these areas was evaluated for success of endosseous implants placed in our study.

Christoph H.F. Hammerie et $\mathrm{al}^{8}$ concluded that the survival rate of implant in regenerated bone using barrier membranes varied between $79 \%$ and $100 \%$, with the majority of the studies indicating more than $90 \%$ success after at least 1 year of function.

PA Fugazzotto et $\mathrm{al}^{9}$ demonstrated a success rate of $96.7 \%$ in implants in previously augmented mandibular bone or bone regenerated by guided bone regeneration.

Similarly M.E Fritz and Abdulwassie et al ${ }^{10,11}$ concluded that implant placed in regenerated bone shows the same clinical radiological and histomorphometric characteristics as those placed in normal bone.

This is in concurrence with the present study where we have placed 15 implants in regenerated bone. Clinical and radiographic assessments of the implant sites showed $93.3 \%$ osseointegration. The observations found in this study indicate that the procedure can be effective in regenerated bone holding a promise for patients in whom bone defects are created due to disease conditions like cysts and tumors.

\section{References}

[1]. Martins WD, Ávila LFC. Partial Spontaneous Bone Regeneration Subsequent to Mandibulectomy. J Contemp Dent Pract 2004 August;(5)3:108-120.

[2]. PA Fugazzotto. Success and failure rates of osseointegrated implants in function in regenerated bone for 72 to 133 months.Int $\mathrm{J}$ Oral Maxillofac Implants, January 1, 2005; 20(1): 77-83.

[3]. Kova'cs AF. Clinical analysis of implant losses in oral tumor and defect patients. Clin Oral Impl Res 2000: 11: 494-504.

[4]. Kim BO, Hong KS, Kim SG. Finite element analysis of cylinder type implant placed into regenerated bone with type IV bone quality. J Korean Assoc Oral Maxillofac Surg. 2004 Aug;30(4):331-338.

[5]. Matteo Chiapasco,Alessandro Rossi,Jason Jones Motta ,Michele Crescentini. Spontaneous Bone Regeneration After Enucleation of Large Mandibular Cysts:A Radiographic Computed Analysis of 27 Consecutive Cases. J Oral Maxillofac Surg .2000;58:942-948.

[6]. Dr. Philip J. The restoration of resected mandibles in children without the use of bone grafts. , Head \& Neck Surgery 6,( 2) ,626631

[7]. Ahmed M. A. Hyperbaric oxygen results in an increase in rabbit calvarial critical sized defects Oral Surg Oral Med Oral Pathol Oral Radiol Endod 2006;101:144-9

[8]. Hammerle CHF ,Jung RE, Feloutzis A :A Systematic review of the survival of implants in bone sites augmented with barrier membranes(guided bone regeneration ) in partially edentulous patients.J Clin Periodontol $2002: 29(3): 226-231$.

[9]. PA Fugazzotto Success and failure rates of osseointegrated implants in function in regenerated bone for 6 to 51 months: a preliminary report.

Int J Oral Maxillofac Implants, January 1, 1997; 12(1): 17-24.

[10]. M.E.Fritz ,M.K Jeffcoat,M.Reddy,D.Koth,L.D Braswell J.Malmquist,J.Lemons. Implants in regenerated bone in a primate model. J.Periodontol. $2001 ; 72: 703-708$

[11]. Abdulwassie, Hassan, Dhanrajani, P. J. Prosthodontic Implant Rehabilitation After the Treatment of a Pathologic Lesion in the Mandible: A Case Report. Implant Dentistry. 2001 10(3):178-181.

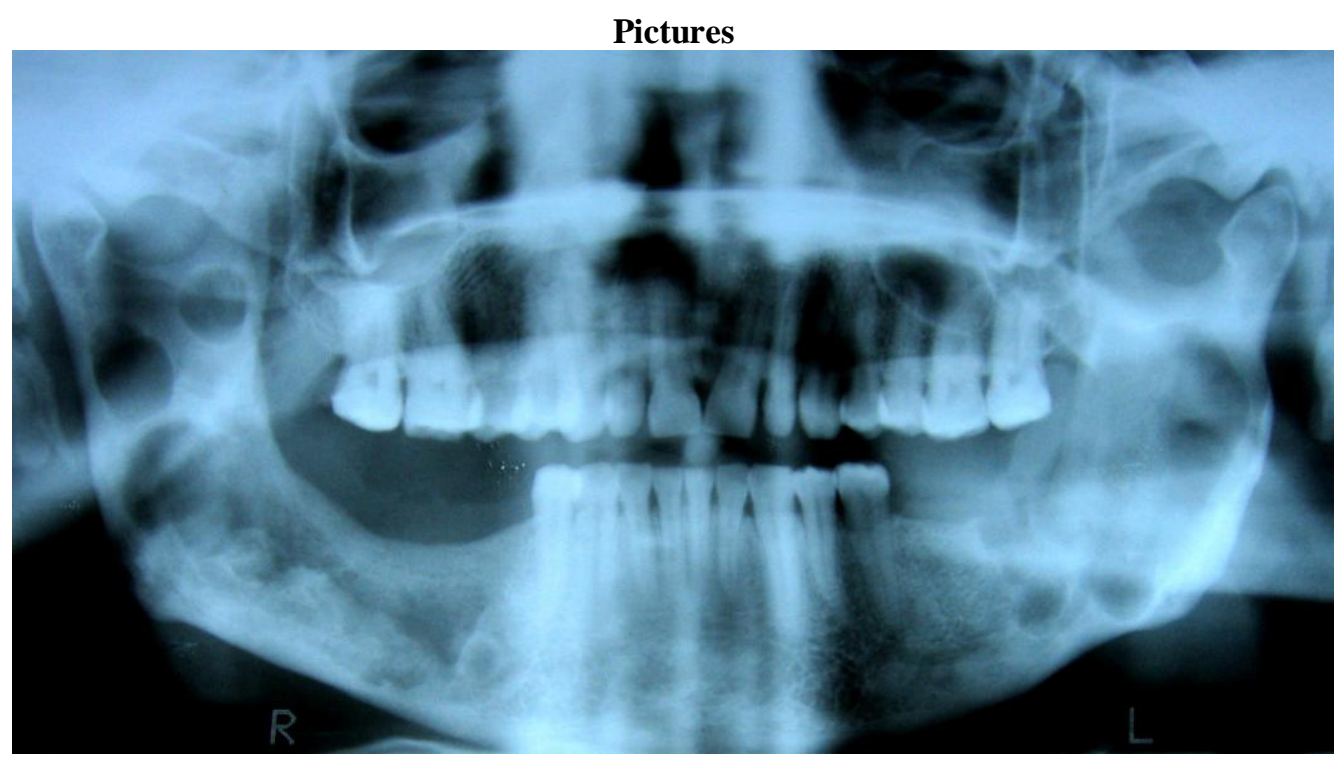

Fig 1. Pre-Operative OPG showing multilocular odontogenic keratocyst 


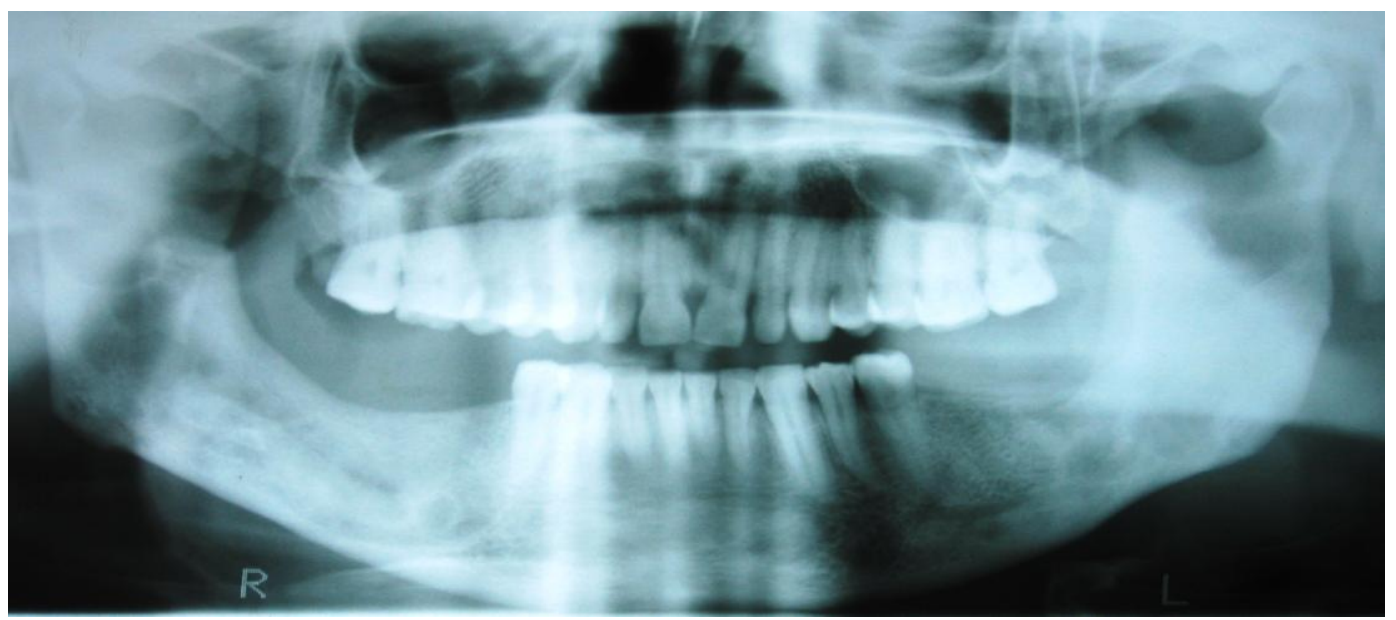

Fig 2. OPG after 1yr of successful enucleation

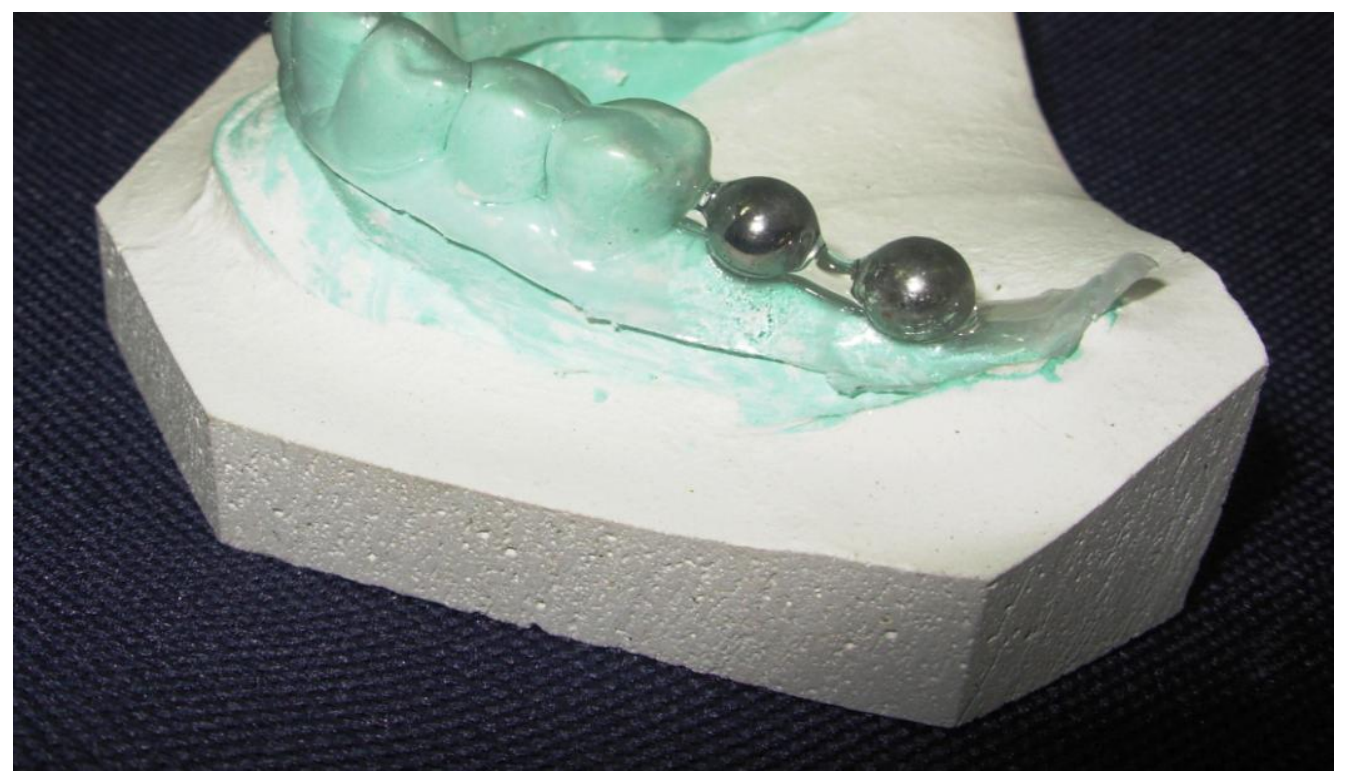

Fig 3. Calculation of distortion in OPG for planning size of implant

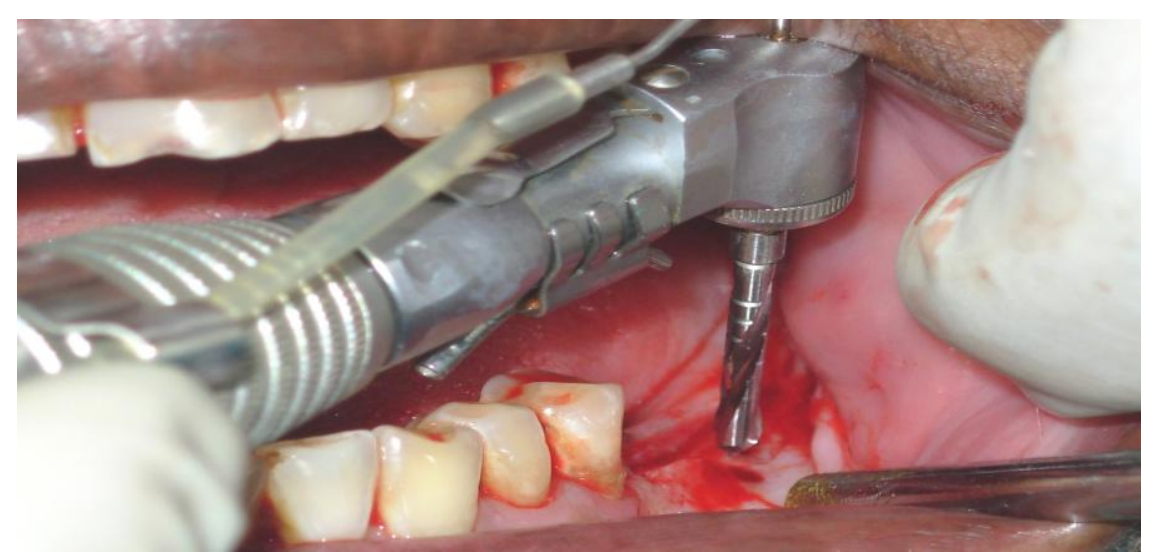

Fig 4. Implant placement in the surgical site 


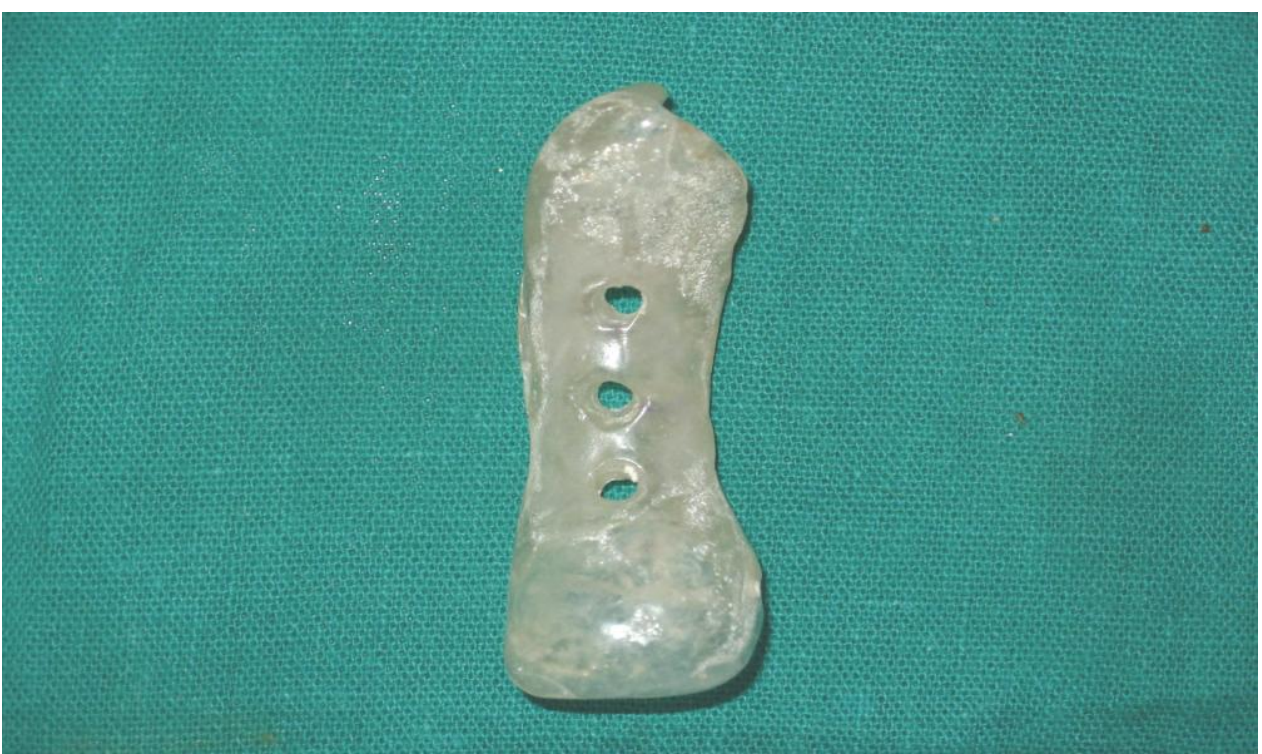

Fig 5. Surgical stent for lower left posterior region

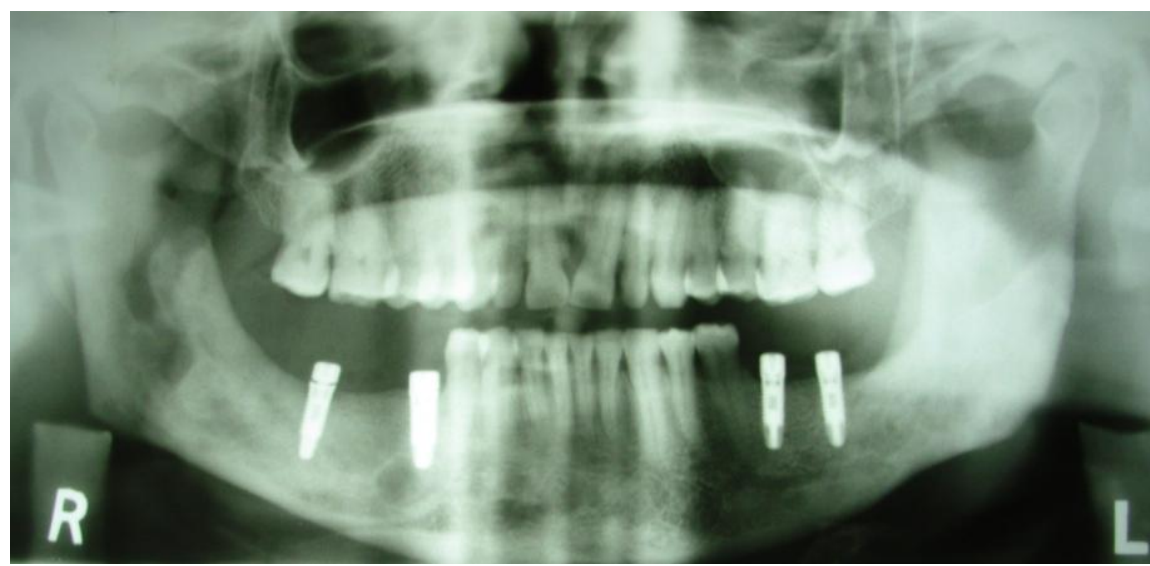

Fig 6. OPG showing implants six months post-insertion

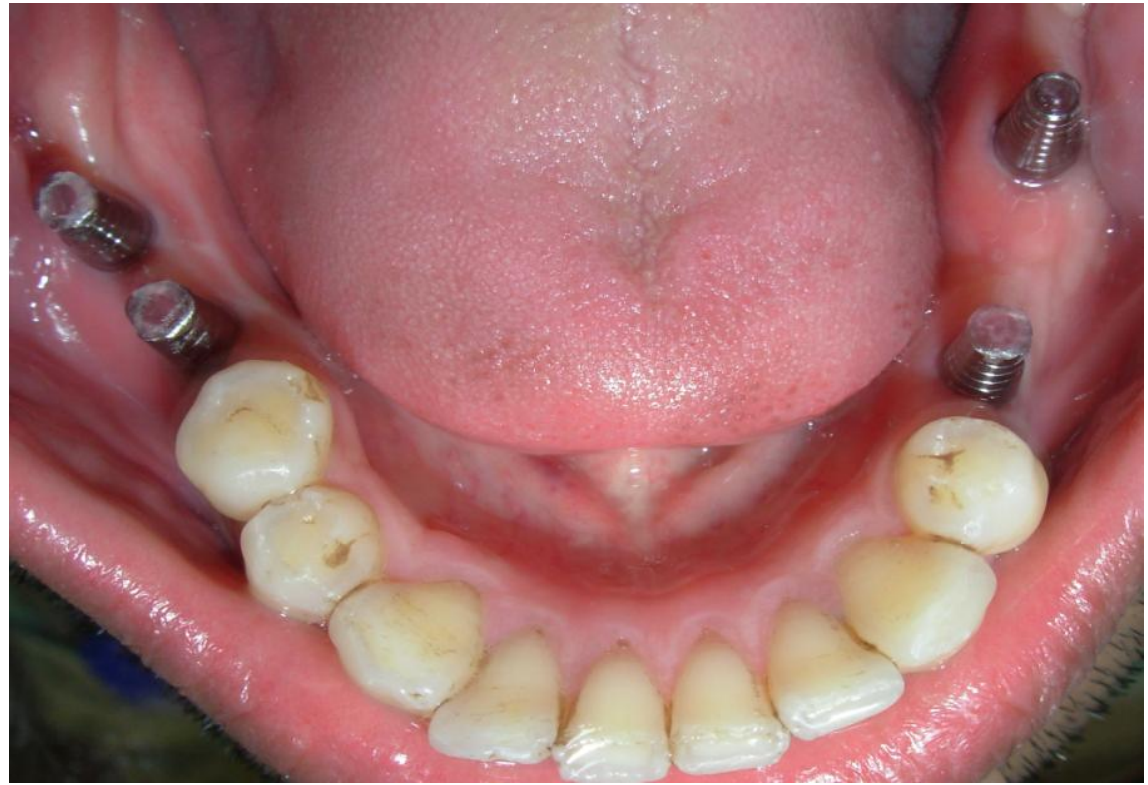

Fig 7. Abutments placed over osseointegrated implants 